\title{
Utilización de recursos diagnósticos y terapéuticos en pacientes ingresados por insuficiencia cardíaca: influencia del servicio de ingreso (estudio INCARGAL)
}

\author{
Use of Diagnostic and Therapeutic Resources in Patients Hospitalized for Heart \\ Failure: Influence of Admission Ward Type (INCARGAL Study)
}

\author{
Alberto García Castelo, Javier Muñiz García, Pascual Sesma Sánchez y Alfonso Castro \\ Beiras, en representación del grupo de estudio INCARGAL
}

\begin{abstract}
Resumen
Antecedentes. La insuficiencia cardíaca es la enfermedad cardiológica de más crecimiento en las naciones desarrolladas, y supone ya la primera causa de ingreso en ancianos. No se ha estudiado bien la diferencia que el servicio de ingreso supone en cuanto al manejo de la insuficiencia cardíaca ni los factores que condicionan el servicio de ingreso.

Objetivos. Establecer si existen diferencias de manejo pronóstico en función del servicio de ingreso (cardiología frente a medicina interna y geriatría) en pacientes con insuficiencia cardíaca.

Pacientes y método. Estudio transversal en que 951 pacientes (505 varones y 446 mujeres) ingresados consecutivamente por insuficiencia cardíaca en los servicios de cardiología $(n=364)$, medicina interna y geriatría $(n$ = 587) de 14 hospitales de Galicia fueron reclutados durante un período máximo de 6 meses, registrándose en el momento del ingreso las principales variables epidemiológicas y clínicas, complicaciones, tratamientos y situación en el momento del alta.

Resultados. Los pacientes con insuficiencia cardíaca tenían una edad media de 75,5 $\pm 12,4$ años $(78,5 \pm 10,6$ en mujeres y 72,7 $\pm 13,5$ en varones). La estancia media fue de $11 \pm 8$ días, con un 50,8\% de primeros ingresos, siendo la mortalidad global hospitalaria del 6,8\%. El 58,9\% de los pacientes tenía hipertensión arterial, el 31,8\% cardiopatía isquémiea, el $27,7 \%$ valvulopatía, el 28,4\% diabetes mellitus y el 32,5\% EPOC. Por servicios, los pacientes atendidos en servicios de cardiología son más jóvenes $(72,5 \pm 13,3$ frente a 77,4 $\pm 11,4$ años; $p<0,005)$, con más varones $(51,9$ frente a 3,7\%; $\mathrm{p}<0,01)$, mayor proporción de primeros ingresos (54,8 frente a 48,4; $\mathrm{p}<0,05)$ Y de edema agudo de pulmón $(22,8$ frente a 9,2\%; P < 0,001). Las odds ratio (y sus intervalos de confianza [IC] del 95\%) de realización de procedimientos diagnósticos y terapéuticos en función del servicio de ingreso (el grupo de referencia es medicina interna-geriatría), ajustando por edad, sexo, función sistólica, número de ingresos y antecedentes personales de demencia, hipertensión arterial, EPOC, infarto agudo de miocardio, valvulopatía, arteriopatía periférica y cardiopatía isquémica, son: ecocardiograma, 3,31 (2,42-4,52); cateterismo, 6,61 (2,78-15,73); ingreso en UCI, 3,4 (1,48-7,8); revascularización, $2,93(0,54-15,74)$, y tratamiento con bloqueado res beta $2,87(1,37-6,04)$. No se observaron diferencias en la mortalidad temprana $(6,6 \%$ en cardiología frente a $7 \%$ en medicina interna-geriatría) ni en la estancia media.

Conclusiones. El servicio de ingreso determinó una clara diferencia en el manejo de la insuficiencia cardíaca, con una mayor adhesión a los protocolos de tratamiento y uso de recursos por parte de los cardiólogos que no se tradujo en diferencias en la mortalidad temprana. Se precisa un seguimiento de los pacientes para evaluar el impacto de estas diferencias en el pronóstico y la evolución de la insuficiencia cardíaca a medio y largo plazos, así como la relación coste-beneficio en una población de edad media avanzada.
\end{abstract}

\footnotetext{
Abstract

Background. Heart failure (HF) is the most rapidly growing cardiac pathology in industrialized countries, and already the primary cause of hospital admissions of elderly people. Outside the field of clinical trials, there have not been many studies in Spain of the influence of the admission department on diagnostic and therapeutic management, whether this affects short-term and long-term prognosis, and the factors that determine the department the patient is admitted to.

Objectives. To analyze whether management and prognosis of patients admitted with heart failure differ depending on the admission ward (cardiology versus internal medicine-geriatrics).

Patients and method. Cross-sectional study of 951 patients (505 men and 446 women) consecutively hospitalized for $\mathrm{HF}$ in the cardiology $(\mathrm{n}=363)$ and internal medicine-geriatrics $(\mathrm{n}=588)$ wards of 12 hospitals of Galicia and recruited over a maximum period of 6 months. The main epidemiological and clinical variables were recorded at admission, and the complications, treatments, and clinical status were recorded at release.
} 
Results. HF patients had a mean age of $75.5 \pm 12$ years (women 78.5 years and men 72.6 years). The average hospitalization time was $11 \pm 8$ days and $50.8 \%$ were first admissions. Total hospital mortality was $6.8 \%$. Fifty-nine percent $(58.9 \%)$ of patients had arterial hypertension, $31.9 \%$ ischemic heart disease, $27.6 \%$ cardiac valve disease, $28.5 \%$ diabetes mellitus, and $32.5 \%$ chronic obstructive pulmonary disease (COPO). The patients admitted to cardiology ward were younger $(72.5 \pm 13$ vs $77.4 \pm 11$ years; $\mathrm{p}<0.005)$, more frequently men (51.9 vs $43.7 \%$; $\mathrm{P}<$ $0.005)$, more often first hospitalizations ( 54.8 vs $48.4 \%$; $\mathrm{P}<0.005)$, and acute pulmonary edema was more common (22.8 vs $9.2 \%$; $\mathrm{P}<0.005$ ). The odds ratio (and 95\% CI) for therapeutic and diagnostic procedures in relation to admission ward (reference group internal medicine-geriatrics), adjusted for age, sex, systolic function, number of hospitalizations, and history of dementia, hypertension, COPO, AMI, valve disease and ischemic heart disease, are: echocardiogram, 3.49 (2.58-4.73); catheterization, 6.42 (3.29-12.55), admission to intensive care, 3.94 (2.15-7.25), revascularization, 2.15 (0.57-8.08), and beta-blocker treatment, 3.39 (1.93-5.97). No differences in hospital mortality (6.6\% in cardiology vs $7 \%$ in internal medicine-geriatrics) or average hospitalization time were found between departments.

Conclusions. The admission ward was related with a clear difference in HF management, with better adherence to guidelines and more use of resources by cardiologists. This was unrelated with differences in hospital mortality so a longer follow-up of these patients is required to evaluate the impact of these therapeutic measures on the prognosis and evolution of $\mathrm{HF}$, as well as the cost-benefit relation in an elderly patient population.

Palabras clave: Insuficiencia cardíaca. Tratamiento. Procedimientos diagnósticos. Cardiología. Medicina interna.

Key words: Heart failure. Treatment. Diagnostic procedure. Cardiology. Internal medicine.

\author{
Abreviaturas \\ IAM: infarto agudo de miocardio. FA: fibrilación auricular. \\ UCI: unidad de cuidados intensivos. EAP: edema agudo de pulmón. HTA: hipertensión arterial. \\ EPOC: enfermedad pulmonar obstructiva crónica. ACV: accidente cerebrovascular. \\ ECG: electrocardiograma. \\ IECA: inhibidor de la enzima convertidora de la angiotensina. \\ FE: fracción de eyección. IC: intervalo de confianza.
}

\title{
Introducción
}

La insuficiencia cardíaca se ha convertido en un gran problema de salud pública en los últimos años ${ }^{1}$, siendo en la actualidad el motivo más frecuente de hospitalización en nuestro país en pacientes mayores de 75 años $^{2}$. Es el síndrome que complica un gran número de enfermedades cardíacas en estadios avanzados de la enfermedad, con una prevalencia creciente debida, entre otras causas, al envejecimiento de la población y a los avances en el tratamiento de la cardiopatía isquémica, que han traído consigo una mejora de la supervivencia de los pacientes con infarto agudo de miocardio (IAM). En las naciones desarrolladas es la única enfermedad cardíaca en la que está aumentando la prevalencia y la mortalidad total-". Todo esto ocasiona un incremento creciente en la carga asistencial, sobre todo hospitalaria, por insuficiencia cardíaca. Por otra parte, ha cambiado su perfil clínico, al afectar a una población de pacientes con características diferentes a los de décadas pasadas, con mayores edad y comorbilidad. Se trata, además, de una complicación grave y progresiva, con una mortalidad del $50 \%$ a los 5 años, similar a la mortalidad media de todos los cánceres ${ }^{3}$.

Existe poca información recogida de forma sistemática sobre cómo se maneja la insuficiencia cardíaca en el ámbito asistencial, es decir, en una población de pacientes no seleccionada ${ }^{9-13}$ que puede diferir de la que se incluye en los principales ensayos clínicos, así como el grado de aplicación de tratamientos aceptados ${ }^{14-18}$ que se deriva de los resultados de estos ensayos clínicos ${ }^{19-30}$. Esta cuestión es interesante ya que, en los últimos 10 años, la incorporación a las guías de diagnóstico y tratamiento de los nuevos conocimientos en la fisiopatología de la insuficiencia cardíaca, la mejora y mayor acceso a procedimientos diagnósticos y terapéuticos, así como la aparición de tratamientos farmacológicos que disminuyen la mortalidad por insuficiencia cardíaca, como los inhibidores de la enzima convertidora de la angiotensina (IECA) y los bloqueadores beta, no parecen haber trasladado esta mejora en el pronóstico, observada en los ensayos clínicos, a la población general atendida por insuficiencia cardíaca ${ }^{31}$.

Existen pocos datos en la bibliografía respecto a uno de los factores que parece incidir en un diferente manejo de la insuficiencia cardíaca, como el tipo de servicio en que ingresa el paciente, es decir, cardiología frente a medicina interna y geriatría. Recientemente, un estudio italiano ha abordado el problema ${ }^{12}$ y se ha levantado una controversia en la comunidad médica acerca de qué clínicos deben ser los encargados del tratamiento de la insuficiencia cardíaca, controversia de la que se ha hecho eco la 
Sociedad Española de Cardiología en un reciente editorial ${ }^{32}$, que generó un número muy elevado de cartas al director.

En este trabajo se analiza el manejo hospitalario de la insuficiencia cardíaca en la comunidad gallega y las diferencias entre especialistas de cardiología e internistas-geriatras (que son los médicos más implicados en el tratamiento de la insuficiencia cardíaca hospitalaria), aspecto de interés con potenciales implicaciones prácticas y del que no se dispone de información en nuestro país.

El objetivo puede concretarse en responder a la pregunta sobre si el hecho de ser tratado por diferentes especialistas (cardiología frente a internistas y geriatras) determina un manejo hospitalario distinto de la insuficiencia cardíaca.

\section{Pacientes y método}

El estudio INCARGAL (INsuficiencia CARdíaca en GALicia) es un estudio transversal, realizado en servicios seleccionados de los 14 hospitales públicos de la red del SER GAS en Galicia, en el que se estudió a pacientes ingresados de forma consecutiva con el diagnóstico principal de insuficiencia cardíaca durante un período de muestreo máximo de 6 meses. Se incluyó a 951 pacientes en esos 14 hospitales (de los centros con población asignada, no participaron dos hospitales comarcales que son fundaciones y un hospital privado concertado). En función del tamaño y la actividad previa de cada centro, se asignó (sin exceder el plazo máximo de 6 meses desde el inicio de la recogida de la muestra) la inclusión de 50, 100 o 150 pacientes con el diagnóstico principal en ese ingreso de insuficiencia cardíaca, admitidos en los servicios participantes (cardiología, medicina interna y geriatría en dos de los centros). No se incluyó a pacientes que no llegaron a ser ingresados en planta por fallecimiento o alta desde el servicio de urgencias. A todos los participantes se les solicitó, mediante consentimiento informado, su aceptación a ser incluidos en el estudio, mantener sus datos en soporte informático y, eventualmente, ser contactados para seguimiento. En el desarrollo del protocolo del estudio se incluyó una reunión entre los investigadores para consensuar una definición sindrómica de insuficiencia cardíaca ${ }^{14-16}$ de utilización habitual en la clínica. Se aceptó el diagnóstico clínico de los investigadores responsables (cardiólogos, médicos internistas y geriatras) sin realizar ninguna comprobación de la uniformidad de la utilización de los diagnósticos, con el objeto de que los pacientes reflejasen mejor la práctica asistencial, de forma que los enfermos incluidos en el estudio constituyen un compendio de lo que los clínicos reconocen como insuficiencia cardíaca en la práctica clínica.

Durante el ingreso, los investigadores participantes recogieron información directamente del paciente (o, en su defecto, de familiar cercano) conforme a un cuestionario estructurado: variables sociodemográficas (edad, sexo, estado civil, situación laboral), antropométricos (se pesó y talló a los pacientes), antecedentes personales de factores de riesgo, antecedentes clínicos y tratamiento farmacológico ambulatorio en el momento del ingreso, tipo de éste (primer ingreso o no), clínica de presentación fundamental y factores precipitantes, caracterización de la insuficiencia cardíaca (izquierda o global), y se completó con los datos proporcionados por el médico responsable referentes al manejo diagnóstico y terapéutico del paciente durante su ingreso hospitalario y en el alta. Se registraron las complicaciones durante el ingreso y la situación en el momento del alta. Se intentaba establecer siempre una causa etiológica fundamental de la insuficiencia cardíaca y, en conexión con esto, el diagnóstico de cardiopatía isquémica se basaba en la presencia de IAM previo, o la presencia de test que demostrase isquemia miocárdica o presencia de lesiones coronarias.

El tamaño de la muestra $(n=951)$ permite una precisión de $\pm 2 \%$, con un intervalo de confianza (IC) del 95\% para una estimación de prevalencia del 50\% (p. ej., proporción de primeros ingresos). La fase de recogida de la muestra se inició en enero de 1999.

\section{Análisis estadístico}

La gestión y el análisis de datos se realizaron de manera centralizada. Para la descripción de variables categóricas se presentan porcentajes y la media y su desviación estándar (DE) para las variables continuas. La comparación entre grupos de las variables continuas se realizó mediante comparación de medias con el test de la t de Student y, para variables cualitativas, el contraste de hipótesis se llevó a cabo mediante el test de la $\chi^{2}$. El error alfa aceptado para todos los contrastes de hipótesis fue del 0,05 y éstos fueron bilaterales. Se utilizó regresión logística para estimar la odds ratio (OR) y su IC del $95 \%$ de realización de diversas intervenciones diagnósticas y terapéuticas en función del servicio de ingreso, ajustado por aquellas covariables que podrían tener una influencia independiente en la variable dependiente a estudio: edad, sexo, antecedentes de demencia, IAM, enfermedad pulmonar obstructiva crónica (EPOC), valvulopatía, cardiopatía isquémica e hipertensión arterial (HTA), presencia de edema 
agudo de pulmón (EAP) y tipo de ingreso (primer ingreso o ingresos previos), variables cuya presencia en el modelo fue forzada por su probable repercusión en la variable dependiente (test diagnóstico o tratamiento) en función de su relevancia clínica. Cada OR e IC del 95\% resultan de un modelo de regresión logística. Por último, se realizó un análisis de regresión con el ingreso en uno u otro servicio como variable dependiente y la inclusión de 13 variables en el modelo predictivo. Se utilizó para el análisis el paquete estadístico de SPSS, versión 9.0.

\section{Resultados}

Se incluyó a 951 pacientes, $505(53,2 \%)$ varones y $446(46,8 \%)$ mujeres, con una media de edad de $75,5 \pm 12,4$ años: 78,5 $\pm 10,6$ años las mujeres y 72,7 $\pm 13,5$ años los varones. El 7,8\% de los pacientes tenía < 60 años; el 17,7\% tenía entre 60 y 69 años, el 36,4\% entre 70 y 79, el 31,7\% entre 80 y 89 , y el 6,4\% más de 90 años. El 50,8\% de los pacientes $(n=486)$ ingresó por primera vez por insuficiencia cardíaca, en tanto que $466(49,2 \%)$ tenía ya algún ingreso previo. La estancia media de ingreso fue de 11 \pm 8 días, con una mediana de 9 días. El 38,2\% de los pacientes $(n=364)$ ingresó a cargo de los servicios de cardiología, mientras que el $61,8 \%(\mathrm{n}=587)$ lo hizo en servicios de medicina interna y geriatría. En la tabla 1 se exponen las principales características de la población a estudio, globalmente considerada y por servicio de ingreso.

Tabla 1. Variables demográficas y clínicas de la población de estudio al ingreso y en función del servicio de ingreso (cardiología frente a medicina interna y geriatría)

\begin{tabular}{|c|c|c|c|c|}
\hline Varones & 43,7 & 51,9 & 0,01 & 53,2 \\
\hline Primer ingreso & 48,4 & 54,8 & 0,05 & 50,8 \\
\hline Estancia (días) $(\mathrm{X} \pm \mathrm{DE})$ & $10,8 \pm 8,1$ & $11,4 \pm 8,1$ & 0,27 & $11 \pm 8$ \\
\hline Consumo etanol > 60 g/día & 4,7 & 11,8 & 0,05 & 7,5 \\
\hline Hipertensión arterial & 61,7 & 54,4 & 0,03 & 58,9 \\
\hline Dislipemia & 17,6 & 24,2 & 0,016 & 20,1 \\
\hline Diabetes mellitus & 29,5 & 26,3 & 0,298 & 28,4 \\
\hline Arteriopatía periférica & 17,0 & 9,1 & $<0,001$ & 14 \\
\hline EPOC & 35,9 & 26,9 & $<0,001$ & 32,5 \\
\hline Arritmias & 29,5 & 34,1 & 0,150 & 31,3 \\
\hline IAM & 13,5 & 16,8 & 0,187 & 14,7 \\
\hline Fibrilación auricular-flúter & 54,7 & 48,1 & 0,04 & 52,2 \\
\hline Onda Q-IAM en ECG & 13,5 & 17,6 & 0,09 & 15 \\
\hline Cardiomegalia & 90,8 & 88,5 & 0,24 & 89,9 \\
\hline $\mathrm{FE}<35 \%(\mathrm{n}=490)$ & 21,5 & 29,5 & 0,04 & 25,7 \\
\hline Cardiopatía isquémica por test positivo & 36,5 & 40,3 & 0,242 & 37,9 \\
\hline Shock & 1,9 & 3 & 0,273 & 2,3 \\
\hline Edema agudo de pulmón & 9,2 & 22,8 & $<0,001$ & 14,4 \\
\hline Insuficiencia cardíaca izquierda & 33,7 & 46,7 & $<0,001$ & 38,7 \\
\hline Mortalidad hospitalaria & 7 & 6,6 & 0,895 & 6,8 \\
\hline
\end{tabular}

Todos los valores se expresan en porcentaje, excepto que se especifique.

EPOC: enfermedad pul manar obstructiva crónica; ACV: accidente cerebrovascular; Onda-Q IAM: ondas Q de infarto en el electrocardiograma; FE: fracción de eyección.

En cuanto a la prevalencia de factores de riesgo, sólo 4,6\% de los pacientes se declaraba fumador activo, siendo 24,8\% $(\mathrm{n}=236)$ el porcentaje de ex fumadores. El 28,4\% era diabético, el 20,1\% tenía hipercolesterolemia y el 58,9\% presentaba HTA, que se configura como el factor de riego de insuficiencia cardíaca más frecuente en nuestra población. Un 7,5\% de los pacientes presentaba una ingesta enólica mayor de $60 \mathrm{~g}$ diarios. La frecuencia de cardiopatía isquémica era del 31,9\% $(\mathrm{n}=303)$ mientras que el $14,8 \%(n=141)$ tenía antecedentes de IAM, el 27,6\% (n = 263) presentaba algún tipo de valvulopatía, un 
32,5\% ( $\mathrm{n}=309)$ estaba catalogado de EPOC y un 7,5\% $(\mathrm{n}=71)$ tenía clínica de demencia. Los síntomas de presentación más frecuentes eran la disnea $(95,6 \%)$, el edema $(52,5 \%)$, dolor torácico $(24,2 \%)$ y la oliguria $(19,9)$. En los pacientes a los que se practicó ecocardiograma durante su ingreso $(\mathrm{n}=490)$, un 25,7\% presentaba disfunción sistólica severa (fracción de eyección [FE] < 35\%), Y un 42,7\% una FE > $50 \%$. Por sexos, cabe destacar una mayor proporción de varones con disfunción ventricular severa $(35,7$ frente a $16,7 \% ; \mathrm{p}<0,005)$.

Los pacientes ingresados en servicios de medicina interna-geriatría y en cardiología constituyen, por tanto, poblaciones de pacientes distintas (tabla 1), con una diferencia estadísticamente significativa en su edad media, 5 años mayor en los servicios de medicina interna-geriatría, que presentan, además, una mayor frecuencia de mujeres y fibrilación auricular (FA). Por el contrario, en los servicios de cardiología se observa un porcentaje mayor de primeros ingresos, de varones y valvulopatías. Es de destacar que no se encontraron diferencias en la estancia media, en la mortalidad, en la prevalencia de IAM previo ni de cardiopatía isquémica evidenciada por tests diagnósticos.

En la tabla 2 se exponen las diferencias en función del servicio de ingreso en el uso de procedimientos diagnósticos y terapéuticos más habituales. Se aprecia una sensible diferencia en el manejo diagnóstico y terapéutico de los pacientes con insuficiencia cardíaca según ingresen en un servicio o en otro, con un empleo mayor de medios diagnósticos, como el ecocardiograma y el cateterismo cardíaco, así como terapéuticos: mayor ingreso en unidad de cuidados intensivos (UCI) y empleo de bloqueadores beta y de indicaciones de revascularización coronaria por parte de los cardiólogos. En la tabla 3 se presentan, estratificadas en función de primer ingreso o ingresos previos, las diferencias entre servicios en la frecuencia de realización de ecocardiograma y cateterismo cardíaco. Tanto en uno como en otro estrato se realizan con mayor frecuencia estas pruebas en los servicios de cardiología.

Tabla 2. Uso de procedimientos diagnósticos y terapéuticos en función de servicio de ingreso

\begin{tabular}{lccc}
\hline Manejo diagnóstico/terapéutico & $\begin{array}{c}\text { Medicina interna-geriatría } \\
(\mathrm{n}=587)\end{array}$ & $\begin{array}{c}\text { Cardiología } \\
(\mathrm{n}=364)\end{array}$ & $\mathrm{p}$ \\
\hline Espirometría & 5,5 & 6,9 & 0,37 \\
Ecocardiograma & 40,6 & 73,8 & $<0,001$ \\
Cateterismo & 2 & 17,6 & $<0,001$ \\
Ingreso UCI & 3,1 & 16,8 & $<001$ \\
lECA & 59,9 & 59 & 0,80 \\
Bloqueadores beta & 3,9 & 13 & $<0,001$ \\
Revascularización & 0,7 & 2,7 & 0,010 \\
Traslado otro centro & 2,7 & 4,1 & 0,18 \\
Trasplante & 0 & 0,5 & 0,07 \\
\end{tabular}

Todos los valores expresados en porcentaje a menos que se especifique.

IECA: inhibidores de la enzima convertidora de la angiotensina; UCI: unidad de cuidados intensivos.

Tabla 3. Porcentajes de realización de ecocardiograma y coronariografía por tipo de servicio, estratificado por primer ingreso o existencia de ingresos previos

\begin{tabular}{|c|c|c|c|c|}
\hline & Medicina interna-geriatría & Cardiología & Global & $\mathrm{p}$ \\
\hline \multicolumn{5}{|l|}{ Ecocardiograma } \\
\hline Primer ingreso & $\begin{array}{c}51,8 \\
(146 / 282)\end{array}$ & $\begin{array}{c}82,4 \\
(164 / 199)\end{array}$ & $\begin{array}{c}64,4 \\
(310 / 481)\end{array}$ & $<0,001$ \\
\hline Ingresos previos & $\begin{array}{c}30,6 \\
(92 / 301)\end{array}$ & $\begin{array}{c}62,8 \\
(103 / 164)\end{array}$ & $\begin{array}{c}41,9 \\
(195 / 465)\end{array}$ & $<0,001$ \\
\hline \multicolumn{5}{|l|}{ Coronariografía } \\
\hline Primer ingreso & $\begin{array}{c}2,1 \\
(6 / 282)\end{array}$ & $\begin{array}{c}22,1 \\
(44 / 199)\end{array}$ & $\begin{array}{c}10,4 \\
(50 / 481)\end{array}$ & $<0,001$ \\
\hline Ingresos previos & $\begin{array}{c}2 \\
(6 / 302)\end{array}$ & $\begin{array}{c}12,2 \\
(20 / 164)\end{array}$ & $\begin{array}{c}5,6 \\
(26 / 466)\end{array}$ & $<0,001$ \\
\hline
\end{tabular}

Entre paréntesis se expresan los números en que está basado cada porcentaje.

En cuatro pacientes no se registró adecuadamente la historia de ingresos previos y en uno la realización o no de ecocardiograma 
En la tabla 4 se exponen las OR de uso de diferentes pruebas di agnósticas en función del servicio de ingreso después de ajustar por todas aquellas variables que se consideró podrían influir en la frecuencia de uso de éstas, como se indica en el apartado Pacientes y método.

Tabla 4. Odds ratio (OR) de realización de diversas pruebas y tratamientos en función del servicio de asistencia (medicina internageriatría frente a cardiología), ajustado por edad, sexo, edema agudo de pulmón, función sistólica, existencia de ingresos previos y antecedentes personales de demencia, EPOC, IAM, ACV, valvulopatía, arteriopatía periférica, cardiopatía isquémica y HT A

\begin{tabular}{|c|c|c|c|}
\hline Procedimiento diagnóstico-terapéutico & $\mathrm{OR}^{*}$ & IC del $95 \%$ de la OR & $\mathrm{p}$ \\
\hline \multicolumn{4}{|l|}{ Durante el ingreso } \\
\hline Espirometría & 1,04 & $0,50-2,16$ & 0,92 \\
\hline Ecocardioqrama $^{a}$ & 3,31 & $2,43-4,52$ & $<0,001$ \\
\hline Cateterismo $^{\mathrm{b}}$ & 6,61 & $2,78-15,73$ & $<0,001$ \\
\hline Revascularización ${ }^{\mathrm{b}}$ & 2,93 & $0,54-15,74$ & 0,21 \\
\hline Ingreso en UCI & 3,40 & $1,48-7,80$ & $<0,001$ \\
\hline \multicolumn{4}{|l|}{ Al alta } \\
\hline Bloqueadores beta ${ }^{b}$ & 2,87 & $1,37-6,04$ & 0,005 \\
\hline $\mathrm{IECA}^{\mathrm{b}}$ & 1,27 & $0,85-1,90$ & 0,23 \\
\hline Espironolactona $^{\mathrm{b}}$ & 1,02 & $0,75-1,93$ & 0,43 \\
\hline
\end{tabular}

Grupo de referencia, medicina interna-geriatría. Una OR > 1 indica una mayor probabilidad de realización de la prueba en los pacientes atendidos por cardiología en comparación con los de medicina interna-geriatría.

${ }^{a}$ aSe excluyó la covariable función sistólica del análisis de regresión de la variable dependiente «realización de ecocardioqrarna» porque la función sistólica expresada en FE fue estimada mediante dicha prueba. ${ }^{\mathrm{b}} \mathrm{N}=886$, número de pacientes vivos a los que se indica tratamiento al alta. IECA: inhibidores de la enzima convetidora de la angiotensina; UCI: unidad de cuidados intensivos.

En la tabla 5 se exponen los resultados del modelo de análisis multivariado mediante regresión logística de aquellos factores que podrían tener relevancia en la decisión de ingreso de un paciente en uno u otro servicio, tomando como referencia el ingreso en el servicio de medicina interna-geriatría solamente en aquellos centros donde había la posibilidad de optar entre ambos tipos de servicios.

Tabla 5. Determinantes de ingreso en cardiología en un análisis multivariado exploratorio de regresión logística

\begin{tabular}{lccc}
\hline Variable & OR & IC del 95\% de la OR & p \\
\hline & & & $<0,001$ \\
Edad & 0,96 & $0,95-0,98$ & 0,028 \\
AP IAM & 1,69 & $1,06-2,71$ & 0,008 \\
AP valvulopatía & 1,61 & $1,13-2,28$ & 0,09 \\
AP HTA & 0,75 & $0,54-1,05$ & 0,006 \\
AP EPOC & 0,62 & $0,44-0,88$ & 0,009 \\
AP demencia & 0,40 & $0,20-0,79$ & 0,002 \\
APACV & 0,47 & $0,29-0,76$ & 0,075 \\
FA-flúter & 0,75 & $0,54-1,03$ & $<0,001$ \\
EAP & 2,51 & $1,60-3,94$ & \\
\end{tabular}

AP: antecedentes personales; IAM; infarto agudo de miocardio; HTA: hipertensión arterial; EPOC: enfermedad pulmonar obstructiva crónica; ACV: accidente cerebrovascular; FA-flúter: fibrilación auricular-flúter auricular; EAP: edema agudo de pulmón. Se considera en el análisis sólo a los pacientes que ingresan en los centros en que han participado servicios de los dos tipos considerados. Además de las variables que permanecen, en el modelo completo se incluyeron sexo, ingresos previos y antecedentes personales de cardiopatía y de diabetes, que se retiraron por no aportar nada sustancial al modelo final. 


\section{Discusión}

Se realizó una reunión para unificar criterios diagnósticos pero, debido a que el diagnóstico de insuficiencia cardíaca es eminentemente clínico, es posible que existan diferencias entre los centros en su aplicación. Aunque en ocasiones puede constituir un problema ${ }^{33}$, creemos que en este caso es más bien un punto fuerte del estudio, en el sentido de que los criterios de inclusión de pacientes favorecen que la población estudiada sea reflejo de la práctica clínica habitual y mejora las posibilidades de generalizar el estudio dentro del entorno en el que se ha realizado. De los 14 hospitales participantes en el estudio, tres son hospitales terciarios con servicio de cardiología, centro de referencia para hemodinámica, electrofisiología y cirugía cardíaca, con docencia y formación de residentes de cardiología, en tanto que en general los demás son hospitales con servicio de medicina interna y cardiología, con docencia (pero no de especialistas en cardiología y, en 2 centros, con servicio de geriatría). Alguno de los hospitales participantes no tienen servicio de cardiología independiente del servicio de medicina interna.

En un análisis global de la muestra destaca, en primer lugar, la edad avanzada, con una media de 75 años, superior a la de los pacientes incluidos en los principales ensayos clínicos de insuficiencia cardíaca. Por otra parte, la proporción de mujeres $(46,8 \%)$ fue notablemente mayor a la de la mayoría de los ensayos clínicos sobre insuficiencia cardíaca, que incluyen menos del $30 \%$ de mujeres ${ }^{20,21}$, en parte debido a su mayor edad. La primera reflexión es que, al igual que se observó en otros estudios ${ }^{12}$, los ensayos clínicos de insuficiencia cardíaca estudian a poblaciones muy diferentes a las atendidas por los clínicos.

La baja tasa de mortalidad hospitalaria $(6,8 \%, 65$ fallecimientos de 951 ingresados) coincide con los datos de la bibliografía que hacen referencia a un descenso de mortalidad por insuficiencia cardíaca ${ }^{2}$, lo cual parece debido a un aumento de frecuencia de esta afección por fallo diastólico, asociado a un mejor pronóstico y que contribuye a este aumento de la prevalencia, al cronificarse una enfermedad asociada hasta hace poco a una elevada mortalidad. En este estudio, el $42,7 \%$ de los pacientes a los que se realizó un ecocardiograma tenía una FE normal, proporción mayor a otras series, que refieren un porcentaje entre un 15 y un $30 \%$ de pacientes con síntomas clásicos de insuficiencia cardíaca con FE normal ${ }^{34,35}$. A este respecto, el factor de riesgo de insuficiencia cardíaca más frecuente en este trabajo es la HTA, presente en el $60 \%$ de los casos. La mortalidad en nuestro estudio es, además, muy similar a la observada $(5,6 \%)$ en un estudio previo realizado en un hospital de los participantes en el INCARGAL ${ }^{13}$.

Como segundo punto diferencial, los pacientes atendidos por cardiólogos frente a los de internistasgeriatras tenían un perfil clínico diferente. Por servicios (tabla 1), los pacientes que ingresaron a cargo del servicio de cardiología eran más jóvenes, con una mayor proporción de varones, y se trataba de un primer ingreso, así como una mayor prevalencia de valvulopatías y de insuficiencia cardíaca izquierda. En cambio, los pacientes atendidos por internistas y geriatras eran de mayor edad, con una mayor proporción de mujeres, de insuficiencia cardíaca congestiva y de pacientes con ingresos previos por insuficiencia cardíaca, sin diferencias en la tasa de cardiopatía isquémica o IAM; también presentaban una mayor prevalencia de comorbilidad (accidente cerebrovascular [ACV], demencia, EPOC y arteriopatía).

Por lo que respecta al manejo, en el análisis univariado (tabla 2) no hubo diferencias en el uso de IECA, pero sí una prescripción mucho mayor de bloqueadores beta por parte de los cardiólogos. A pesar de ello, la proporción es baja en ambos casos. No se ha realizado un análisis de la adecuación de los tratamientos y, además, estos datos reflejan la situación presente hace 3 años, pero parece que hay un amplio margen de mejora, especialmente en el tratamiento con bloqueadores beta.

Se observó también una clara diferencia en lo que respecta a un uso más frecuente de ecocardiograma, cateterismo cardíaco, revascularización coronaria e ingreso en la UCI en los pacientes atendidos en los servicios de cardiología. La diferencia en la frecuencia de utilización de ecocardiografía y cateterismo entre los servicios está presente tanto en los pacientes en los que se trataba del primer ingreso como en los demás (tabla 3). A pesar de las diferencias indicadas en el manejo, no se registraron diferencias en la estancia media entre servicios (tabla 1).

Las diferencias en el manejo de los pacientes entre cardiólogos e internistas podría ser debida exclusivamente por ese diferente perfil de los pacientes. Las mayores edad y comorbilidad en los pacientes de medicina interna condicionarían que éstos fueran objeto de un manejo más conservador y con un menor uso de recursos diagnósticos y terapéuticos. Esto no se confirma al realizar el análisis multivariado individualizado para cada variable de diagnóstico y tratamiento, ajustando por aquellas covariables que podrían tener una influencia en la variable dependiente a estudio (tabla 4). Después de ajustar por todas estas variables, el servicio donde el paciente ingresa permanece como una variable independiente que condiciona, en general, un mayor uso de recursos diagnósticos y terapéuticos en los servicios de cardiología. Estas variaciones en el tratamiento no se acompañan de diferencias detectables en la mortalidad intrahospitalaria global, si bien la tasa de mortalidad hospitalaria fue relativamente baja. Por otra parte, los beneficios de las diferencias de manejo, de existir, serían a más largo plazo. 
Se investigó qué determina que un paciente ingrese en uno u otro servicio, ya que parece que este hecho es el que condiciona de forma más importante el manejo y tratamiento de la insuficiencia cardíaca. En este análisis sólo se incluyeron los centros en los que había opción posible (cardiología o medicina interna-cardiología). Se intentó identificar los factores independientes que motivan que un paciente ingrese en un servicio de un tipo u otro, encontrándose en el análisis multivariado para las covariables estudiadas (tabla 5) que los únicos factores independientes que favorecían el ingreso en cardiología fueron presentar una valvulopatía, haber tenido un IAM previo e ingresar en EAP, en tanto que la edad avanzada, la EPOC, haber tenido un ACV, presentar FA o flúter y la demencia favorecen hacerlo en medicina interna. Por el contrario, el sexo del paciente no influye a la hora de decidir el ingreso en diferentes servicios, sin apreciarse en este aspecto un agravio del tipo síndrome de Yentl, descrito en otras enfermedades ${ }^{35-39}$.

Aunque los datos disponibles en la bibliografía son escasos, se ha podido constatar, en al menos un estudio de seguimiento clínico en un hospital terciario de los EE.UU. con programa de trasplante ${ }^{40}$, que el uso de recursos terapéuticos invasivos, como cateterismo, angioplastia, implante de marcapasos o desfibriladores, cirugía de revascularización, reemplazo valvular y trasplante cardíaco en la población de pacientes que ingresan por insuficiencia cardíaca, se ha incrementado significativamente en el decenio 1986-1996, especialmente en el trienio 1994-1996, asociándose este mayor intervencionismo con un descenso de la razón de mortalidad estandarizada (mortalidad observada/mortalidad prevista), y de la mortalidad hospitalaria ajustada, junto con una reducción en la estancia media. Al mismo tiempo no se observó un aumento de los costes desde el bienio 1990-1991, en que se produjo el máximo nivel de gasto no ajustado, disminuyendo a partir de entonces. Parece que tras un aumento en los costes iniciales, a medio plazo, la estrategia invasiva no sólo salva vidas, sino que también puede ser rentable para el sistema de salud desde un punto de vista de coste-beneficio.

Este estudio no proporciona un seguimiento que permita evaluar el impacto en el pronóstico a medio plazo en los pacientes de estas diferencias en el manejo de la insuficiencia cardíaca, por lo que serían precisos estudios adicionales. Sin embargo, las diferencias existen y son de gran magnitud y su corrección, si se considera necesaria, deberá implicar a profesionales diversos.

\section{Conclusiones}

La insuficiencia cardíaca en nuestro medio es una enfermedad que afecta a pacientes de edad media avanzada con una mortalidad hospitalaria temprana no demasiado elevada, lo que puede estar en relación con la elevada proporción de insuficiencia cardíaca con función sistólica normal. Los médicos internistas y geriatras atienden a pacientes con insuficiencia cardíaca de más edad que los cardiólogos, y también a una mayor proporción de mujeres que éstos. Atender a más mujeres no se debe a sexismo en la selección de pacientes, sino a su mayor edad. Las mujeres constituyen alrededor de la mitad del total de pacientes con insuficiencia cardíaca atendidos en los hospitales, porcentaje superior a los principales ensayos clínicos sobre insuficiencia cardíaca. Existe una diferencia clara en el tratamiento de la insuficiencia cardíaca por servicios. Los cardiólogos utilizan con más frecuencia los bloqueadores beta y el intervencionismo diagnóstico y terapéutico, sobre todo en la cardiopatía isquémica. Esto no se traduce en una diferente mortalidad hospitalaria temprana, pero podría implicar un pronóstico más favorable y una mejor calidad de vida futura para el paciente. Es conveniente evaluar este aspecto en un seguimiento ulterior, así como la carga asistencial futura para el sistema de salud, lo que permitirá valorar el costebeneficio de las nuevas medidas terapéuticas en la insuficiencia cardíaca en esta población de edad avanzada.

\section{Centros e investigadores participantes en el proyecto INCARGAL}

Investigador principal: Alfonso Castro Beiras.

Centro coordinador: ODDS, S.L.: Javier Muñiz García y Alberto García Castelo.

Hospital Juan Canalejo. A Coruña. Servicio de Cardiologia:

José Ángel Rodríguez Fernández, Beatriz Bouzas Zubeldia, Ignacio Mosquera Pérez y Lorenzo Monserrat Iglesias. Servicio de Medicina Interna: Vicente Ramos Polledo, Fernando de la Iglesia Martínez, Ricardo Nicolas Miguel y Carlos Pellicer Váiquez.

Hospital Arquitecto Marcide. Ferro!. Servicio de Medicina Interna: Pascual Sesma Sánchez y Carlos González González.

Hospital Clínico Universitario. Santiago. Servicio de Medicina Interna: Vicente Lorenzo Zúñiga, María Rosario Alende Sixto, Carmen Mella Pérez, Emilio Manuel Padín paz y lago Villamil Cajato. 
Hospital De Conxo. Santiago. Servicio de Medicina Interna: Antonio Pose Reino. Hospital XeralCalde. Lugo. Servicio de Cardiología: Juan Vidán Martínez y Ricardo Izquierdo González. Servicio de Xeriatria: Fernando Veiga Fernández, José Ramón Martínez Calvo y Manuel Melero Brezo.

Hospital Comarcal De Burela. Lugo. Servicio de Cardiología: José Antonio Lombán Villanueva.

Hospital Comarcal de Monforte. Servicio de Cardiología: Nicolás Bayón Meleiro.

Hospital Santa Maria Nai. Orense. Servicio de Cardiologia: Miguel Pérez de Juan Romero, María Dolores Collell Mach y Rosa Mojón Perezy. Servicio de Medicina Interna: Manuel de Toro Santos, Arnalia Cadavid Rodríguez, Serafín Pérez Pombo, Justa Rego Villa Amor, Miguel Ángel Rodríguez Quintela y Elvira Rodríguez Torres.

Hospital Cristal Piñor. Ourense. Servicio de Cardiologia: Evaristo Freire Castroseiros. Servicio de Medicina Interna: Ovidio Fernández y Manuel Jiménez Martínez.

Hospital Comarcal de Valdeorras. Servicio de Medicina Interna: Josep Masferrer Serra yA. Eneriz Calvo.

Hospital Xeral-Cíes. Vigo. Pontevedra. Servicio de Cardiología: Hugo Torrealday Taboada y José Penas Cortés. Servicio de Medicina Interna: Bernardo Sopeña López.

Hospital Do Meixoeiro. Servicio de Cardiología: M. Victoria Platero Vázquez, E. Martín y Estrella Pérez Fernández. Servicio de Medicina Interna: José Carlos Medraño Martínez. Servicio de Geriatría: Carlos Rodríguez Pascual, M." Luz López Blanco y M. Teresa Olloz Chiva.

Hospital Montecelo de Pontevedra. Servicio de Cardiologia: Juan Raúl Casariego Rosón, Rodrigo Medina Alba.Benito Puen te Rodero y Pedro Vigil Escalera.

Hospital Provincial de Pontevedra. Servicio de Cardiología; Manuel Silva Martínez, Concepción Fernández Costa y M." Jose Pedros Cuadrillero. Servicio de Medicina Interna: Antonio Martínez Muradas.

\section{Bibliografía}

1. Sociedad Española de Cardiología. Estudio de los recursos, necesidades y organización para la atención al paciente cardiológico. Madrid: Sociedad Española de Cardiología, 1999.

2. Rodríguez Artalejo F, Guallar-Castillón P, Banegas Banegas JR, del Rey Calero J. Trends in hospitalization and mortality for heart failure in Spain, 1980-1993. Eur HeartJ 1997;17:71-9.

3. Ho KKL, Pinsky JL, Kannel WB, Levy D. The epidemiology of heart failure: the Framingham Study. J Am ColI Cardiol 1993; 22(Supl A):6-13.

4. Gillum RF. The epidemiology of heart failure In the United Stateso Am Heart J 1993; 14: 1158-62.

5. Ghali JK, Cooper R, Ford E. Trends in hospitalization rates for heart failure in the United States, 1973-1986. Evidence for increasing population prevalence. Arch lntern Med 1990; 150:769-73.

6. Yamani BM, Massie BM. Congestive Heart Failure: Insights from epidemiology, implications for treatment. Mayo Clin Proc 1993; 68: 1214-8.

7. Foot DK, Lewis RP, Pearson TA, Beller GA. Demographics and cardiology, 1950-2050. J Am Coll Cardiol 2000;35(Suppl B):66B-80B.

8. McMurray JJV, Petrie MC, Murdoch DR, Davie AP. Clinical Epidemiology of Heart failure, public and private health burden. Eur Heart J 1998; 19(5uppl):9-16.

9. Havranek EP, Abrams F, Stevens E, Parker K. Determinants of mortality in elderly patients with heart failure: the role of angiotensin-converting enzyme inhibitors. Arch Intern Med 1998; 158: 2024-8.

10. Gambassi G, Forman DE, Laplane KL, Mor V, Sgadari A, Lipsitz LA, et al. Management of heart failure among very old persons living in long-term care: has the voice of trials spred? The SAGE Study Group. Am Heart J 2000; 139:85-93.

11. Croft JB, Giles WH, Polard RA, Keenan NL, Casper ML, Anda RF. Heart failure survival among older adults in the United States: a poor prognosis for an emerging epidemic in the Medicare population. Arch lntern Med 1999; 159:505-10.

12. Bellotti P, Badano LP, Acquarone N, Griffo R, Lo Pinto G, Maggioni AP, et al. Specialty-related differences in the epidemiology, clinical profile, manage and outcome patients hospitalized for heart failure. The OSCUR study. Eur Heart J 2001;22: 596-604.

13. Ramos Polledo V, Pita Fernández S, de la Iglesia Martínez F, Pellicer Vázquez C, Nicolás Miguel R, Diz-Lois Martínez F: et al. Etiología, características clínicas, causa desencadenante, tipo de disfunción ventricular, estancia media y mortalidad de 305 pacientes ingresados por insuficiencia cardíaca. An Med Interna (Madrid) 2000; 17: 19-24.

14. Guidelines for evaluation and management of heart failure. Report of the American College of Cardiology/American Heart Association Task Force on Practice Guidelines (Committe on evaluation and management of heart failure). Circulation 1995;92: 2764-84.

15. Cohn JN. The management of chronic heart failure. N Engl J Med 1996;335:490-8.

16. Navarro-López F, de Teresa E, López-Sendón JL, Castro-Beiras A, y Grupo de Trabajo de insuficiencia Cardíaca de la Sociedad Española de Cardiología. Guías del diagnóstico, clasificación y tratamiento de la IC y del shock cardiogénico. Rev Esp Cardiol 1999;52(Supl 2): 1-49. 
17. Goldsmith SR, Dick C. Differentiating systolic from diastolic heart failure: pathophysiologic and therapeutic considerations. Am J Med 1993;95:645-55.

18. Califf RM, Bengtson JR. Cardiogenic shock. N Engl J Med 1994;330: 1724-30.

19. The SOLVD Investigators. Effect of enalapril on survival in patients with reduced left ventricular ejection fractions and congestive heart failure. N Engl J Med 1991 ;325:293-330.

20. The SOLVD investigators. Effect of enalapril on mortality and development of the heart failure in asymptomatic patients with reduced ejection fractions. N Engl J Med 1992;327:685-91.

21. Pfeffer MA, Braunwald E, Moye LA, Basta L, Brown El Jr, Cuddy TE, et al, on behalf of the SAVE Investigators. Effect of captopril on mortality and morbidity in patients with left ventricular dysfunction after myocardial infarction: results of the Survival and Ventricular Enlargement trial. N Engl J Med 1992;327:669-77.

22. The Acute lnfarction Ramipril Efficacy (AIRE) Study Investigators. Effect of ramipril on mortality and morbidity of survivors of acute myocardial infarction with clinical evidence on heart failure. Lancet 1993;342:821-8.

23. Kober L, Torp-Pedersen C, Carlsen JE, Bagger H, Eliasen P, Lyngborg K, et al. A clinical trial of the angiotensin-converting-enzyme inhibitor trandolapril in patients with left ventricular dysfunction after myocardial infarction. Am J Cardiol 1994;73:44C-50C.

24. Packer M, Gheorghiade M, Young JB, Constantini PJ, Adams KF, Cody RJ, et al. Withdrawal of digoxin from patients with chronic heart failure treated with angiotensin-converting enzyme Inhibitors: RADIANCE Study. N Engl J Med 1993;329: 1-7.

25. Packer M, Bristow MR, Cohn JN, Colucci WS, Fowler MB, Gilbert EM, et al. The effect of carvedilol on morbidity and mortality in patiens with chronic heart failure. N Engl J Med 1996; 334: 1349-55.

26. Pitt B, Martínez FA, Meurers G, Cowley AJ, Thomas 1, Deedwania PC, et al, on behalf of ELITE Study investigators. Randomized trial on losartan versus captopril in patients over 65 with heart failure (Evaluation of Losartan in the Elderly Study, ELITE). Lancet 1997;349:747-52.

27. The CONSENSUS Trial Study Group. Effects of enalapril with hydralazine-issosorbide dinitrate in the treatment of chronic congestive heart failure. N Engl J Med 1987;316: 1429-35.

28. The CIBIS lnvestigators and Committees. A randomized trial on betablockade in heart failure. The Cardiac Insufficiency Bisoprolol Study. Circulation 1994;90:1765-73.

29. Packer M, O'Connor CM, Ghali JK, Pressler ML, Carson PE, Belkin RN, et al. Effect of amlodipine on morbidity and mortality in severe chronic Heart Failure. The Prospective Randomized Amlodipine Survival Evaluation Study Group. N Engl J Med 1996;335:1107-14.

30. Kostis JB, Rosen RC, Cosgrove NM, Shindler DM, Wilson AC. Non pharmacological therapy improves functional capacity and emotional status in congestive heart failure. Chest 1994; 106:996-1001.

31. Maclntyre K, Capewell S, Stewart S, Chamers JWT, Boyd J,. Finlayson A, et al. Evidence of improving prognosis in heart failure. Trends in case fatality in 66547 patients hospiralized between 1986 and 1995. Circulation 2000; 102: 1 126-31.

32. Anguita Sánchez M, Vallés Belsué F. ¿Quién debe tratar la insuficiencia cardíaca? Rev Esp Cardiol 2001;54:8158 .

33. Alonso-Pulpón L. La epidemia de insuficiencia cardíaca: ¿son todos los que están y están todos los que son? Rev Esp Cardiol 2002;55:211-4.

34. Bonow RO, Udelson JE. Left ventricular diastolic dysfunction as a cause of conzestive heart failure. Ann lntem Med 1992; 117:502-9.

35. Dougherty AH, Naccarelli GV, Gray EL, Hicks CH, Goldstein RA. Congestive heart failure with normal systolic function. Am J Cardiol 1984;54:778-82.

36. Shumaker SA, Brooks MM, Schron EB, Hale C, Kellen re, Inkster M, et al. Gender differences in health-related quality of life among postmyocardial infarction patients: brief report. CAST Investigators. Cardiac Arrhythmia Suppression Trials. Women's Health 1997;3:53-60.

37. Larsen JA, Kadish AH. Effects of gender on cardiac arrhythmias. J Cardiovascular Electrophysiol 1998;9:655-64.

38. Demirovic J, Blackburn H, McGovern PG, Luepker R, Sprafka JM, Gilbertson D. Sex differences in early mortality after acute myocardial infarction (The Minnesota Heart Survey). Am J Cardiol 1995;75:1096-101.

39. Hendel RC. Myocardial infarction in women. Cardiology 1990; 77(SuppI2):41-57.

40. Polanczyk CA, Rohde LE, Dec GW, DiSalvo T. Ten-year trends in hospital care for congestive heart failure: improved outcomes and increase use of resources. Arch lntern Med 2000;160:325-32. 